\title{
The Evolution of $\mathrm{H} \alpha$ Profiles in S-type Mira Stars
}

\author{
A.W. Woodsworth \\ Dominion Astrophysical Observatory, National Research Council, Victoria, Canada
}

Past attempts to model the $\mathrm{H} \alpha$ emission profiles from Mira stars have been hampered by the strong absorption bands produced by $\mathrm{TiO}$ (M-type Miras) or carbonbased molecules (C-type Miras). The S-type Miras, however, exhibit relatively clean emission lines. As part of a larger study of Mira variables, I have obtained a series of high-dispersion $\mathrm{H} \alpha$ profiles of S-type Miras at different phases, using the DAO 48" coudé spectrograph. Although most profiles were obtained from different stars, their shapes develop in a regular way with advancing phase, so it appears that the observations can be used together to provide a good data base for the fitting of evolutionary (rather than static) models for the shock waves in S-type Miras.

Initial experiments with fitting simple models to the profiles have been extremely encouraging. The profiles can be represented very well by three Gaussian components. The characteristic asymmetry of the blue side of the profile is reproduced, and the stellar velocity obtained from microwave measurements of circumstellar $\mathrm{CO}$ is midway between the red-most component and the new, third component blended with the blue-most component. The accelerations obtained agree with the model of Tuchman (1991), and all the components evolve in a regular way with advancing phase. It is not certain whether these results imply three separate physical components, but they do characterize the line morphology very well and must carry at least some physically meaningful information.

\section{References:}

Tuchman, Y., 1991, Astrophys.J., 383, 779

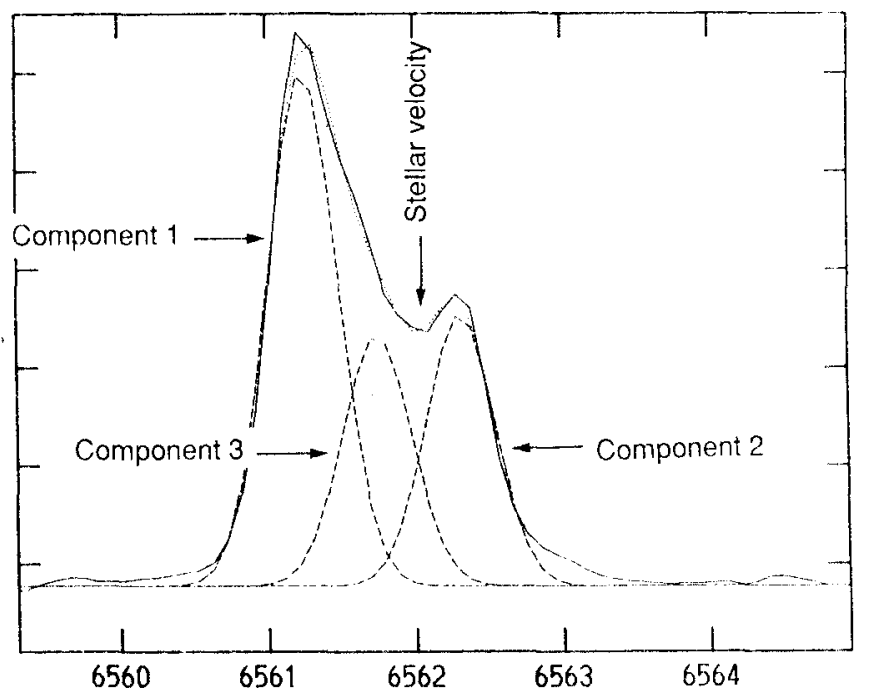

Figure 1 Sample Gaussian fits to S-star $\mathrm{H} \alpha$ emission profiles 